\title{
Zum Stellenwert der Imagination und des Imaginären in neueren
} Fiktionstheorien

Klinkert, Thomas

Posted at the Zurich Open Repository and Archive, University of Zurich ZORA URL: https://doi.org/10.5167/uzh-113993

Journal Article

Accepted Version

Originally published at:

Klinkert, Thomas (2014). Zum Stellenwert der Imagination und des Imaginären in neueren Fiktionstheorien. Kodikas/Code, 37(1/2):55-67. 


\title{
Zum Stellenwert der Imagination und des Imaginären in neueren Fiktionstheorien
}

\author{
Thomas Klinkert (Freiburg i. Br.)
}

\begin{abstract}
This article presents an analysis of three recent theories of fiction, which differ from traditional theories of fiction, by conceiving fiction as a ternary relationship. Instead of construing a binary opposition between fiction and reality, in the manner of traditional theories (Plato), the authors considered here start from the premise that fiction can be theorized by the integration of imagination or the imaginary. Wolfgang Iser and Rainer Warning consider fiction as a kind of discourse, in which the imaginary is dealt with and in which there is a coupling of processes of imagination and knowledge. Whereas Iser follows Aristotle, in saying that in fictional discourse there is a "reformulation of the formulated world", which helps to better understand the world, Warning adopts a Foucaultian perspective, which is combined with the conception of the "radical imaginary" proposed by Castoriadis. Kendall L. Walton defines fiction as a "game of make-believe", based on the transformative power of imagination. In all three conceptions, imagination or the imaginary widen the traditional perspective on fiction as opposed to reality. This presents the advantage of defining fiction positively and not negatively, as a deficient type of discourse.
\end{abstract}

\section{Die binäre Logik traditioneller Fiktionstheorien}

Seit der Antike gibt es Theorien der Fiktion, welche auf der binären Opposition zwischen Wirklichkeit und Erfindung, Urbild und Abbild, Wahrheit und Nicht-Wahrheit beruhen. So begründet Platon bekanntlich im zehnten Buch der Politeia den Ausschluss der Dichter aus dem idealen Staat durch eine Kritik der Mimesis. ${ }^{1}$ Die Maler und Dichter seien Nachahmer von Seiendem. Dadurch stehen sie für Platons Dialogfigur Sokrates noch unter den Werkmeistern, die ihre Gegenstände immerhin als Nachahmung der Ideen erschaffen. Die mimetischen Künstler ahmen dagegen nur diese von der Wahrheit der Ideen bereits entfernten Gegenstände nach, sind also Nachahmer des Nachgeahmten. In beiden Fällen liegt ein UrbildAbbild-Verhältnis vor, mithin eine binäre Opposition. Mimesis wird als scheinhafte, minderwertige Verdoppelung der Wirklichkeit abgewertet. So sagt Sokrates zu Glaukon: "Oder merkst du nicht, daß auch du selbst imstande wärest dies [sc. die sichtbaren Dinge der Schöpfung] auf gewisse Weise hervorzubringen?" und antwortet auf Glaukons Frage: "Und welches wäre diese Weise?" folgendermaßen:

Es hat mit ihr gar keine Schwierigkeit, sondern man hat sie vielfältig und rasch zur Hand; am schnellsten wohl, wenn du ohne Umstände einen Spiegel nimmst und ihn überall herumträgst:

1 Zum Zusammenhang zwischen antiken Mimesis-Theorien und Fiktionstheorien vgl. Petersen (2000). 
alsbald wirst du da eine Sonne machen und was sonst am Himmel ist, alsbald auch eine Erde, alsbald auch dich selbst und die übrigen Geschöpfe, Geräte, Gewächse und alles vorhin Genannte. (Platon 1998: 390 [596 d])

Wie derjenige, der mithilfe eines Spiegels die Wirklichkeit schlicht verdoppele, sei auch der Maler. Zwar mache auch er ein Bett, aber nur ein scheinbares, wie sich Sokrates und Glaukon schnell einig werden.

Ein solcher auf der binären Opposition von Urbild und Abbild, von Sein und Schein beruhender Fiktionsbegriff beherrscht die Diskussion bis heute. So gilt er beispielsweise im aktuellen deutschen Rechtssystem, wie man anhand des berühmt gewordenen Falles Esra von Maxim Biller zeigen kann. Dieser im Jahr 2003 erschienene Roman erzählt die Liebesgeschichte zwischen Adam und Esra, einem Schriftsteller und einer Schauspielerin. Die Romanhandlung beruht auf einer realen Liebesbeziehung zwischen dem Autor des Textes und einer Frau, die sich in der Figur der Esra wiederzuerkennen glaubte und sich - aufgrund der Preisgabe intimer Details durch ihren ehemaligen Geliebten - in ihrem Persönlichkeitsrecht beeinträchtigt fühlte, weshalb sie vor Gericht ging. Der Rechtsstreit zwischen der ehemaligen Geliebten des Autors und dem Verlag, in dem das Buch erschienen war, wurde in verschiedenen Instanzen geführt und endete vor dem Bundesverfassungsgericht in Form eines Beschlusses des Ersten Senats vom 13. Juni 2007 (Bundesverfassungsgericht 2007).

Dem Beschluss des Verfassungsgerichts sind vier Leitsätze vorangestellt, von denen drei für die dem Urteil zugrunde liegende Auffassung von Fiktionalität besonders aussagekräftig sind:

[...] 2. Die Kunstfreiheit verlangt für ein literarisches Werk, das sich als Roman ausweist, eine kunstspezifische Betrachtung. Daraus folgt insbesondere eine Vermutung für die Fiktionalität eines literarischen Textes. 3. Die Kunstfreiheit schließt das Recht zur Verwendung von Vorbildern aus der Lebenswirklichkeit ein. 4. Zwischen dem Maß, in dem der Autor eine von der Wirklichkeit abgelöste, ästhetische Realität schafft, und der Intensität der Verletzung des Persönlichkeitsrechts besteht eine Wechselbeziehung. Je stärker Abbild und Urbild übereinstimmen, desto schwerer wiegt die Beeinträchtigung des Persönlichkeitsrechts. Je mehr die künstlerische Darstellung besonders geschützte Dimensionen des Persönlichkeitsrechts berührt, desto stärker muss die Fiktionalisierung sein, um eine Persönlichkeitsrechtsverletzung auszuschließen.

Aufschlussreich ist, dass das Bundesverfassungsgericht einen engen Zusammenhang zwischen kunstspezifischer Betrachtung und Fiktionalität postuliert. Fiktionalität fungiert gewissermaßen als Legitimationsinstanz für die Kunst, wobei der Grad der Freiheit, den die Kunst genießt, mit dem Grad ihrer Fiktionalität korreliert. In der Argumentation, die sich in dem Beschluss findet, ist folglich immer wieder die Rede von Übereinstimmungen zwischen der Romanhandlung und der Wirklichkeit. So heißt es beispielweise in den Gründen des Beschlusses unter Punkt A.II.18: "Eine Verselbständigung des Abbilds vom Urbild sei vorliegend nicht zu erkennen. Bis auf die Namen habe der Autor in dem Buch die familiären Beziehungen '1:1' der Wirklichkeit entnommen." In Satz 21 liest man: "Eine genügende Verfremdung des Abbilds vom Urbild fehle."

Man erkennt an dieser juristischen Argumentation sehr deutlich die dichotomische Gegenüberstellung von Urbild (Wirklichkeit) und Abbild (Fiktion), wobei der Grad der Übereinstimmung zwischen Fiktion und Wirklichkeit variieren kann. So wird einerseits von der Möglichkeit einer 1:1-Übereinstimmung ausgegangen, andererseits von der Möglichkeit der Verfremdung bzw. Abweichung von der Wirklichkeit. Die juristische Auseinandersetzung 
bezieht sich im vorliegenden Fall auf den Grad dieser Abweichung. Während der Romanautor Biller in seinem Nachwort eine Übereinstimmung zwischen Fiktion und Wirklichkeit verneint, indem er behauptet, dass alle Figuren seines Romans frei erfunden und Ähnlichkeiten mit lebenden oder verstorbenen Personen nicht beabsichtigt und somit rein zufällig seien, geht seine ehemalige Geliebte als Klägerin dagegen von der Annahme einer weitgehenden Deckungsgleichheit von Fiktion und Wirklichkeit aus und fühlt sich dadurch herabgewürdigt und in ihrem Persönlichkeitsrecht verletzt.

Nicht nur im juristischen Diskurs, sondern auch im Diskurs der Sprachphilosophie lässt sich das Nachwirken eines binären Fiktionsbegriffs nachweisen, der stark an den von Platon definierten erinnert. So begründet der Sprachphilosoph John Searle in einer 1974 erstmals erschienenen einflussreichen und vielzitierten Untersuchung mit dem Titel "Der logische Status fiktionaler Rede" ("The Logical Status of Fictional Discourse") die Eigenart fiktionaler Sprechakte ebenfalls im Rekurs auf eine binäre Opposition, indem er den Wahrheitsbegriff bemüht. Sein Fiktionsbegriff situiert sich im Rahmen der von ihm entwickelten Sprechakttheorie, welche Searle auf folgenden Regeln fundiert:

1. Die wesentliche Regel: Wer etwas behauptet, legt sich auf die Wahrheit der ausgedrückten Proposition fest. 2. Die Vorbereitungsregeln: Der Sprecher muß in der Lage sein, Belege oder Gründe für die Wahrheit der ausgedrückten Proposition anzuführen. 3. Die Wahrheit der ausgedrückten Proposition darf im Äußerungskontext nicht sowohl für den Sprecher als auch für den Hörer offensichtlich sein. 4. Die Aufrichtigkeitsregel: Der Sprecher legt sich auf die Überzeugung fest, daß die ausgedrückte Proposition wahr ist. (Searle 2007: 25)

Ein pragmatischer Sprechakt unterliegt Searle zufolge den genannten Regeln. Ein fiktionaler Sprechakt dagegen kann solche Regeln missachten, wie Searle am Beispiel eines Textauszugs aus einem Roman von Iris Murdoch demonstriert: Zwar sieht der zitierte Textauszug formal aus wie eine Behauptung, wie wir sie etwa auch in einem journalistischen Text finden könnten, doch werden in ihm die für die Behauptung einschlägigen Regeln nicht eingehalten.

Ihre Äußerung legt [sc. Iris Murdoch] nicht auf die Wahrheit der Proposition fest, daß ein kürzlich [...] bestallter Leutnant namens Andrew Chase-White an einem sonnigen Sonntagnachmittag im April 1916 in seinem Garten herumbosselte und dachte, er werde noch zehn herrliche Tage ohne Pferde verbringen. Das mag wahr sein oder nicht - Murdoch ist jedenfalls nicht im geringsten darauf festgelegt, daß es wahr ist. Da sie nicht auf die Wahrheit der Proposition festgelegt ist, braucht sie auch keine Belege für ihre Wahrheit anführen zu können. [...] Da sie nicht darauf festgelegt ist, daß es wahr ist, was sie sagt, stellt sich aber auch die Frage nicht, ob wir davon schon unterrichtet sind oder nicht, und Murdoch wird nicht für unaufrichtig gehalten, wenn sie keinen Augenblick lang wirklich glaubt, daß tatsächlich jemand an jenem Tag in Dublin an Pferde gedacht hat. (Searle 2007: 25)

Es liegt somit ein Sprechakt vor, der aussieht wie eine Behauptung, ohne tatsächlich eine solche zu sein. Searle versucht aus diesem Widerspruch eine Theorie der Fiktion zu entwickeln, die auf dem Prinzip des "Als ob" beruht und folgendermaßen lautet: 1. "Der Autor eines fiktionalen Werkes gibt vor, eine Serie illokutionärer Akte zu vollziehen - normalerweise illokutionäre Akte des behauptenden Typs." (Searle 2007: 27) 2. "Das Kriterium zur Identifikation eines Textes als fiktionales Werk muß notwendigerweise in den illokutionären Intentionen des Autors liegen." (Searle 2007: 28) 3. "Die vorgegebenen Illokutionen, die ein fiktionales Werk konstituieren, werden ermöglicht durch die Existenz einer Menge von Konventionen, welche die Regeln, die normalerweise illokutionäre Akte und Welt zueinander 
in Beziehung setzen, außer Kraft setzen." (Searle 2007: 29) 4. "Die vorgegebenen Vollzüge illokutionärer Akte, die das Schreiben eines fiktionalen Werkes konstituieren, bestehen im tatsächlichen Vollzug von Äußerungsakten, mit der Intention, jene horizontalen Konventionen in Kraft treten zu lassen, welche die gewöhnlichen illokutionären Festlegungen der Äußerungen außer Kraft setzen.” (Searle 2007: 30)

Es kann hier nicht weiter auf die aus literaturwissenschaftlicher Sicht problematische Gleichsetzung von textexternen und textinternen Sprechinstanzen eingegangen werden (vgl. hierzu die Kritik von Genette 1991: 46ff.). Im vorliegenden Zusammenhang wichtig ist die binäre Entgegensetzung von wahren, echten Sprechakten und Pseudo-Sprechakten, als welche fiktionale Sprechakte von Searle klassifiziert werden, d.h. Sprechakten, die nur so tun, als ob sie Behauptungen wären. Die Unterscheidung zwischen 'normalen' und 'nicht-normalen' Sprechakten zeigt an, dass es sich hier um ein Unterordnungsverhältnis handelt. Fiktionale Sprechakte sind eine Besonderheit, die sich für Searle nur als Anomalie beschreiben lässt. Dabei zeigt sich, dass die fiktionalen Sprechakte für ihn ein Problem darstellen, welches er durch argumentative Verrenkungen in den Griff zu bekommen versucht - etwa indem er etwas rätselhaft davon spricht, "jene horizontalen Konventionen in Kraft treten zu lassen, welche die gewöhnlichen illokutionären Festlegungen der Äußerungen außer Kraft setzen”. Mit den Mitteln einer auf binären Oppositionen beruhenden Logik kann man offenbar das Problem, welches fiktionale Sprechakte darstellen, nur schwer lösen.

Die auf der binären Opposition Urbild vs. Abbild beruhende Fiktionsauffassung, wie sie sich sowohl bei Platon als auch bei Searle, aber auch im juristischen Diskurs nachweisen lässt, birgt aus literaturwissenschaftlicher Sicht eine grundlegende Problematik in sich: Wenn es ein Urbild gibt, dann ist stets die Frage, wie sehr das Abbild dem Urbild ähnelt bzw. ob es diesem überhaupt ähnlich sein kann. Mit anderen Worten: Wenn die Fiktion sich an der Wirklichkeit messen lassen muss, dann hat sie es schwer und zieht meist den Kürzeren. So sagt Platon, dass die Mimesis des Dichters oder Malers scheinhaft und unvollkommen sei und in doppeltem Grade von der Wahrheit der Ideen entfernt. Für Searle ist die Frage, wie ein Sprechakt, der aussieht wie ein ernst gemeinter Sprechakt, existieren kann, obwohl für ihn die Wahrheitsbedingungen und Aufrichtigkeitsregeln pragmatischer Sprechakte nicht gelten. Im juristischen Diskurs stellt sich die Frage etwas anders, denn es geht hier bei der Aushandlung des Konflikts zwischen Kunstfreiheit und Persönlichkeitsrecht um die Frage der zulässigen und tolerierbaren Ähnlichkeit zwischen Fiktion und Wirklichkeit bzw. der erforderlichen Abweichung der Fiktion von der Wirklichkeit (Verfremdung). In allen Fällen zeigt sich, dass trotz unterschiedlicher Begründungszusammenhänge das Kernproblem stets die Ähnlichkeitsbeziehung zwischen Fiktion und Wirklichkeit ist und dass die Fiktion gerade durch die Ähnlichkeit ein Skandalon darstellt - entweder weil sie der Wirklichkeit zu wenig ähnlich ist oder weil sie zu wenig von ihr abweicht. Allerdings kann man stets trefflich darüber streiten, ob die Fiktion nun eher der Wirklichkeit ähnelt oder ob sie eher von ihr abweicht. Objektive Kriterien hierfür kann es nicht geben.

Es soll nun im Folgenden argumentiert werden, dass sich durch die Erweiterung der zweistelligen Beziehung zwischen Fiktion und Wirklichkeit, zwischen Urbild und Abbild, in Richtung einer dreistelligen, die Imagination bzw. das Imaginäre einbeziehenden Konstellation neue Perspektiven auf die Fiktion ergeben, die diese in ihrem Eigenrecht und in ihrer Eigengesetzlichkeit positiv fundieren können. Denn auffälligerweise beruhen alle bisher erwähnten Fiktionsbegriffe auf einer (expliziten oder impliziten) negativen Bestimmung; sie sagen, was Fiktion nicht ist: Sie ist nicht die Wirklichkeit, sondern nur ihre Nachahmung, sie ist kein pragmatischer Sprechakt, sondern ein Pseudosprechakt, der so tut, als ob er ein echter 
Sprechakt wäre. Wie zu zeigen sein wird, ermöglicht es die Einbeziehung des Imaginären / der Imagination, Fiktion als einen eigenständigen Bereich zu bestimmen.

\section{Die Erweiterung des binären Modells der Fiktion durch das Imaginäre/die Imagination}

Neben den bisher behandelten 'Standardtheorien' der Fiktion gibt es in jüngerer Zeit Ansätze, die den binären Charakter der Fiktionalität durch die Hinzufügung eines dritten Elements erweitern. Gemeint ist das Element des Imaginären bzw. der Imagination. Drei dieser Ansätze möchte ich im Folgenden diskutieren. Sie stammen von Wolfgang Iser, Rainer Warning und Kendall L. Walton.

\subsection{Wolfgang Iser}

In seinem Buch Das Fiktive und das Imaginäre schreibt Wolfgang Iser: "Nun fragt es sich aber, ob die gewiß handliche Unterscheidung von fiktionalen und nicht-fiktionalen Texten sich an dieser geläufigen Opposition festmachen läßt. Sind fiktionale Texte wirklich so fiktiv, und sind jene, die man so nicht bezeichnen kann, wirklich ohne Fiktionen?" (Iser 1991: 18) Die binäre Opposition von Fiktion und Wirklichkeit - welche zu den "Elementarbeständen unseres 'stummen Wissens"” (Iser 1991: 18) gehöre - stellt Iser im Hinblick auf ihre Tauglichkeit zur adäquaten Beschreibung fiktionaler Texte infrage. In diesen nämlich, so Iser, lassen sich Mischungsverhältnisse von Realem und Fiktivem beobachten. Die zweistellige Relation von Fiktion und Wirklichkeit wird infolgedessen von Iser durch die Triade des Realen, des Fiktiven und des Imaginären ersetzt. "Enthält der fiktionale Text Reales, ohne sich in dessen Beschreibung zu erschöpfen, so hat seine fiktive Komponente wiederum keinen Selbstzweckcharakter, sondern ist als fingierte die Zurüstung eines Imaginären." (Iser 1991: 18)

Iser geht von der wohlbekannten Tatsache aus, dass in fiktionalen Texten Wirklichkeitselemente (z.B. soziale Verhältnisse, aber auch individuelle Gefühle und Empfindungen) dargestellt werden können. Von diesen Wirklichkeitselementen behauptet Iser, sie seien keine Fiktionen und sie würden auch nicht dadurch zu Fiktionen, dass sie in fiktionalen Texten dargestellt würden. Andererseits jedoch gelte auch, dass solche Realitätselemente in fiktionalen Texten nicht um ihrer selbst willen wiederholt würden, d.h. es gehe nicht um bloße Nachahmung oder Abbildung. Er kommt zu dem Schluss, dass im Akt des Fingierens "Zwecke zum Vorschein kommen, die der wiederholten Wirklichkeit nicht eignen." (Iser 1991: 20) Weiter heißt es:

Ist Fingieren aus der wiederholten Wirklichkeit nicht ableitbar, dann bringt sich in ihm ein Imaginäres zur Geltung, das mit der im Text wiederkehrenden Realität zusammengeschlossen wird. So gewinnt der Akt des Fingierens seine Eigentümlichkeit dadurch, daß er die Wiederkehr lebensweltlicher Realität im Text bewirkt und gerade in solcher Wiederholung das Imaginäre in eine Gestalt zieht, wodurch sich die wiederkehrende Realität zum Zeichen und das Imaginäre zur Vorstellbarkeit des dadurch Bezeichneten aufheben. (Iser 1991: 20)

Das Fiktive versteht Iser, wie man sieht, als einen intentionalen Akt der Darstellung; der eigentlich passendere Begriff hierfür wäre 'das Fingieren'. Das Reale, welches dem Akt des Fingierens einerseits gegenübersteht und von ihm abgrenzbar ist, kann andererseits Teil dieses 
Aktes sein; es kann von ihm aufgenommen und zum Teil des Dargestellten werden. Unter dem Realen versteht Iser die außertextuelle Welt, "die als Gegebenheit dem Text vorausliegt und dessen Bezugsfelder bildet" (Iser 1991: 20, Fußnote 2). Konstituiert wird diese außertextuelle Welt unter anderem durch Sinnsysteme, soziale Systeme, Weltbilder, d.h. es handelt sich um einen sehr weiten Begriff vom Realen, in dem auch Texte ihren Platz haben: Das Reale wird von Iser auch bestimmt als "die Vielfalt der Diskurse, denen die Weltzuwendung des Autors durch den Text gilt" (Iser 1991: 20, Fußnote 2). Mit der "Wiederkehr lebensweltlicher Realität" im Akt des Fingierens verbindet sich nun das Imaginäre, und zwar dergestalt, dass die fiktional dargestellte Wirklichkeit zu einem Zeichen wird, welches auf etwas verweist, dessen Vorstellbarkeit, so Iser in einer etwas schwierigen Ausdrucksweise, das Imaginäre ist. Im Akt des Fingierens kommt es somit zu einer gemeinsamen Gestaltwerdung des Realen und des Imaginären. Dieses ermöglicht es, dass man sich etwas vorstellt, es hat Ermöglichungscharakter. Das, was man sich vorstellt, ist Teil einer Zeichenstruktur, d.h. das Imaginäre wird, indem es mit einem Akt des Fingierens interagiert, seinerseits eingebunden in einen semiotischen Zusammenhang.

Isers Begriff des Imaginären ist noch genauer zu untersuchen. Es sei, so sagt er,

in seiner uns durch Erfahrung bekannten Erscheinungsweise diffus, formlos, unfixiert und ohne Objektreferenz. Es manifestiert sich in überfallartigen und daher willkürlich erscheinenden Zuständen, die entweder abbrechen oder sich in ganz anderen Zuständlichkeiten fortsetzen. (Iser 1991: 21)

Mit anderen Worten: Das Imaginäre manifestiert sich in Form von Phantasmen, Projektionen und Tagträumen, die sich durch Nicht-Bestimmtheit auszeichnen. Im Akt des Fingierens werden nun, so Iser, der Bereich des Wirklichen und der Bereich des Imaginären zusammengeschlossen. Es findet dabei eine doppelte Grenzüberschreitung statt. Auf der einen Seite wird die im Fingieren dargestellte Wirklichkeit zum Zeichenhaften hin verändert, wobei ein "Überschreiten ihrer Bestimmtheit" (Iser 1991: 21) erfolgt. Auf der anderen Seite wird das Diffuse und Unbestimmte des Imaginären durch seinen Eintritt in den Akt des Fingierens einer Bestimmtheit zugeführt, die das Imaginäre ursprünglich nicht besitzt. Auf der einen Seite also erfolgt die Überführung einer lebensweltlichen Bestimmtheit des Realen in eine zeichenhafte Bestimmtheit im Rahmen der Fiktion. Auf der anderen Seite findet die Transformation einer Nicht-Bestimmtheit des Imaginären in eine Bestimmtheit im Rahmen der Fiktion statt. Die erste Überführung ist eine Grenzüberschreitung qua Irrealisierung, die zweite eine Grenzüberschreitung qua Realwerden des Imaginären.

Als die Irrealisierung von Realem und Realwerden von Imaginärem schafft der Akt des Fingie-
rens eine zentrale Voraussetzung dafür, inwieweit die von ihm jeweils geleisteten Grenzüber-
schreitungen 1. die Bedingung für die Umformulierung formulierter Welt abgeben, 2. die
Verstehbarkeit einer umformulierten Welt ermöglichen und 3. die Erfahrbarkeit eines solchen
Ereignisses eröffnen. (Iser 1991: 23)

Der Akt des Fingierens beruht also Iser zufolge auf einer doppelten Bewegung, einer doppelten Grenzüberschreitung und einer Inbezugsetzung des Realen und des Imaginären im Medium des Fiktiven. Diese komplexe dreistellige Relation hat, gut aristotelisch gedacht, die Funktion, Weltverstehen zu ermöglichen, indem das Reale neu zusammengesetzt wird und dadurch dem Seienden Alternativmodelle hypothetisch entgegengestellt werden und diese wiederum durch die Fiktion sinnlich erfahrbar gemacht werden können. Im weitesten Sinne ist der Akt des Fingierens also in dieser Dreistelligkeit ein erkenntnisstiftender Vorgang, der konstitutiv angewiesen ist auf den Beitrag des Imaginären. 
Unter Verweis auf verschiedene Theorien des Imaginären - insbesondere auf die Theorien von Coleridge, der Imagination als Vermögen begreift, von Sartre, der das Imaginäre als Vorstellungsakt konzeptualisiert, und von Castoriadis, der vom radikal Imaginären spricht begreift Iser dieses als ein Phänomen, welches fundierenden Charakter besitzt, sich allerdings ursprünglich in einem Außenbereich befindet. Da das Imaginäre selbst unbestimmt ist und nicht bildlich, begrifflich oder anderweitig sich manifestiert, bedarf es der "Mobilisierung von außerhalb seiner, sei es durch das Subjekt (Coleridge), das Bewußtsein (Sartre) oder die Psyche und das Gesellschaftlich-Geschichtliche (Castoriadis)" (Iser 1991: 377). Eine entscheidende Vermittlungsfunktion bei der Aktivierung des Imaginären komme dem Spiel zu. Eine besondere Variante des Spiels sei das Fiktive, welches weniger zweckgerichtet sei als die durch die Namen Coleridge, Sartre und Castoriadis bezeichneten Paradigmen Subjekt, thetisches Bewusstsein und Gesellschaftlich-Geschichtliches. Das Fiktive ziele nicht auf eine Verdinglichung transitorischer Bewegung durch Symbole, es fungiere vielmehr als "Instanz, Imaginäres über seinen pragmatischen Gebrauch hinaus erfahrbar zu machen, ohne von dessen 'Entfesselung' überschwemmt zu werden, wie etwa im Traum oder in Halluzinationen” (Iser 1991: 381). Wichtig ist für Iser die Doppelungsstruktur als distinktives Merkmal des Fiktiven. Er zeigt dies am Beispiel der Bukolik, die "eine artifizielle - weil erfundene Welt mit einer soziohistorischen zusammenschließt und sich in dieser Doppelung als Selbstreflexion literarischer Fiktionalität präsentiert" (Iser 1991: 381). Das Fiktive im Iser'schen Sinne ist somit gekennzeichnet durch Selbstreflexivität und Doppelung und es fungiert als ein Spiel, welches das unvordenkliche Imaginäre ergreift und einer Bestimmtheit zuführt und in diesem Prozess Erkenntnis ermöglicht.

\subsection{Rainer Warning}

Rainer Warning nähert sich der Fiktion zunächst, indem er sie ähnlich wie Searle, allerdings semiotisch sehr viel differenzierter als dieser "über den illokutionären Modus eines Als-obHandelns" (Warning 1983: 191) zu beschreiben versucht. In seinem späteren Beitrag "Poetische Konterdiskursivität. Zum literaturwissenschaftlichen Umgang mit Foucault" (Warning 1999) erweitert er seinen Fiktionsbegriff durch die Einbeziehung des Imaginären. Er stellt die Frage nach dem Status der Literatur im Denken von Michel Foucault und greift dabei den in Les mots et les choses verwendeten Begriff des "contre-discours" (Foucault 1966: 59) auf. Obwohl Foucault seinen Literaturbegriff nur unsystematisch skizziere, scheine, so Warning, die Vermutung nahezuliegen, “daß Foucault poetische Texte grundsätzlich in einem Spannungsverhältnis zu diskursiv organisiertem Wissen, also zur 'Ordnung des Diskurses' sieht, als Freiraum neben und außerhalb von Machtdispositiven” (Warning 1999: 317). Diesen Gedanken aufgreifend, versucht Warning, eine systematischere Fundierung des Konzepts poetischer Nicht- oder Konterdiskursivität vorzunehmen. Dabei macht er deutlich, dass im Sinne Foucaults Literatur keineswegs abzutrennen sei von den gesamtdiskursiven Zusammenhängen. "Literarische Texte in umgreifende Diskursfelder einzubetten ist allein schon deshalb unverzichtbar, weil sie an deren Strukturen, wie vermittelt auch immer, durchaus partizipieren." (Warning 1999: 317) Neben dieser Einbettung aber bleibe doch die "Frage nach der Literarizität von Literatur" (Warning 1999: 318) methodologisch fundiert zu stellen, was Warning mit dem Postulat einer "Dialektik von Einbettung und Ausbettung" (Warning 1999: 318) versucht. 
Im Gegensatz zu Foucaults Affinität zur zeittypischen avantgardistischen Auffassung von der bloßen Selbstreferentialität des Schreibaktes (Tel Quel und Nouveau Nouveau Roman) sieht Warning als “eigentliches Movens poetischer Konterdiskursivität” (Warning 1999: 318) das Imaginäre. Dieses wurde von Foucault selbst in seiner frühen Einführung zu einer Übersetzung von Ludwig Binswangers Traum und Existenz zusammen mit dem Traum "als Offenbarungen letzter Wahrheiten, insbesondere des Todes als Verwirklichung der Freiheit" (Warning 1999: 319) gefeiert. Später jedoch entwickelte Foucault im Rahmen seiner Archäologie des Wissens (Foucault 1966 und 1969) eine Position, in der das Subjekt als problematisches historisches Konstrukt und nicht als Existential konzipiert wurde, weshalb Foucault das Imaginäre nun allenfalls noch als ein Phänomen, welches sich aus Büchern speise, zulassen konnte. So schreibt er über Flauberts Tentation de Saint-Antoine: "L'imaginaire ne se constitue pas contre le réel pour le nier ou le compenser; il s'étend entre les signes, de livre à livre, dans l'interstice des redites et des commentaires; il naît et se forme dans l'entre-deux des textes. C'est un phénomène de bibliothèque." (Foucault 1964: 297f.) Das Imaginäre ist für Foucault also nicht ein an Subjektivität geknüpftes, sondern ein rein diskursives Phänomen.

Um nun aber, so Warning, das Foucault'sche Konzept des Gegendiskurses theoretisch genauer zu fassen, müsse man dem Imaginären Rechnung tragen. Denn so wie Foucault zufolge Wissen sowohl in institutionalisierten Wissensdiskursen als auch in anderen Diskurstypen wie zum Beispiel literarischen Texten seinen Niederschlag finde, sei umgekehrt zu postulieren, dass das Imaginäre ebenfalls Teil aller Diskurse sei, sodass man, so Warning, das Konzept der Episteme überhaupt nicht denken könne, ohne dabei auch den Stellenwert des Imaginären zu berücksichtigen. Um diese Lücke in Foucaults diskursarchäologischem Begriffssystem zu füllen, greift Warning auf das schon bei Iser nutzbar gemachte Konzept des radikal Imaginären von Cornelius Castoriadis zurück. Castoriadis (1975) geht in seiner Theorie des Imaginären aus von Mangel und Begehren. Dies entspricht durchaus dem Denken der Psychoanalyse, mit dem Castoriadis einen kritischen Dialog führt. Während die Psychoanalyse im Rahmen des von ihr entwickelten Modells der Triebdynamik annimmt, dass die phantasmatischen Repräsentationen auf eine Urszene des Mangels zurückgeführt werden könnten, radikalisiert Castoriadis dieses Konzept, indem er den Mangel nicht in einer äußeren Realität, sondern in der Psyche selbst verortet. Die Psyche sei, so Castoriadis, ihr eigenes verlorenes Objekt: "La psyché est son propre objet perdu." (Castoriadis 1975: 433) Der das Imaginäre auslösende Mangel wäre demnach der Psyche selbst eingeschrieben und nicht rückführbar auf eine erlittene Urszene, die ein Therapeut im Dialog mit dem Patienten dingfest machen und dadurch in Wissen überführen könnte. Warning zufolge ergeben sich aus dem Ansatz von Castoriadis zwei Vorteile: Erstens die Verortung des Mangels und Begehrens und des dadurch produzierten Imaginären "außerhalb eines angeblichen Wissens" (Warning 1999: 321). Zweitens die Auffassung des Imaginären als eines Phänomens, das "frei ist von jeder positiven oder negativen Wertung" (ebd.). "Es ist keine Kompromißbildung, es ist keine täuschende Repräsentation, die eine andere verbirgt, es ist keine Sekundärszene, die verweist auf eine Urszene, kurz: Es ist kein Symbol, das der Desymbolisation durch einen Analytiker harrte." (Ebd.)

Wenn nun diese Psyche, die ihren ursprünglichen Mangel in sich selbst hat, zu einem sozialen Individuum wird, dann impliziert dies einen radikalen Bruch der Psyche mit sich selbst. Dennoch bleibt die Psyche "'magnetisiert' durch das, was sie verloren hat, so daß ihre soziale Instituierung nicht einhergeht mit einem Verlust ihrer Kreativität, ihres ständigen Anderswerdens in der Kette der Bilder eines auf immer unstillbaren Begehrens" (ebd.). Dies wiederum bedeutet, dass das radikal Imaginäre, welches die Psyche ursprünglich auszeichnet, 
durchschlägt auf das gesellschaftliche Imaginäre. Das gesellschaftliche Imaginäre steht seinerseits in einer Wechselbeziehung mit dem identitätslogischen Denken des Wissens. Wissen und Imaginäres hängen eng zusammen und durchdringen sich gegenseitig:

Es gibt kein Wissen ohne imaginäre Besetzung, wie es umgekehrt keine Hervorbringung des Imaginären gibt, die nicht Teil des Wissens, die nicht 'gewußt' wäre. Die Episteme und das Imaginäre bilden also eine wesentlich komplexe, eine wesentlich hybride Einheit, und man kann beide folglich nicht trennen über semantische Oppositionen, sondern allein über pragmatische und also funktionale Oppositionen. Man kann mit dem Wissen entweder ernsthaft und diszipliniert umgehen, oder aber mehr zwanglos-spielerisch. [...] Den Wissensdiskurs und den poetischen Konterdiskurs in Opposition setzen ist nicht eine semantische, sondern eine pragmatische Operation. Man kann nicht 'indizieren', was dem Wissen und was dem Imaginären angehört, weil der Konterdiskurs ebenfalls über Organisationsprinzipien, über Formationsregeln verfügt, wie sie etwa die Gattungsregeln darstellen. (Warning 1999: 322)

Durch die Zusammenführung von Castoriadis und Foucault entwickelt Warning somit eine Theorie, in der literarische und nicht-literarische Diskurse in gleicher Weise sowohl am Imaginären als auch am Epistemischen partizipieren. Das Imaginäre wird zur fundierenden Größe, wobei eine Unterscheidung des fiktionalen vom nicht-fiktionalen Diskurs erst durch pragmatische Regeln und Konventionen möglich wird. Diese Auffassung relativiert die in Foucaults Rede vom Konterdiskurs aufscheinende radikale Andersartigkeit der Literatur. Fiktionale Literatur partizipiert auf einer grundlegenden Ebene am Wissen und wird gespeist aus dem Imaginären, genauso wie die auf Wissen spezialisierten Diskurse. Dass auf einer weniger basalen Ebene die Diskurse dennoch unterscheidbar sind, ergibt sich aus Gattungsregeln und pragmatischen Einstellungen der Zeichenbenutzer. Eine wichtige Funktion kommt in diesem Zusammenhang der Räumlichkeit zu, welche Warning mit Lotman als transzendentale Bedingung des Imaginären deutet, dergestalt dass "der poetische Text mit seiner topographischen Konkretisierung der topologischen Achsen die räumliche Arbeit des Traums und des Imaginären gleichsam fortsetzt, wohingegen das Wissen den 'espace onirique' überführt in das räumliche Modell einer gegebenen Gesellschaft oder einer übergreifenden kulturellen Formation." (Warning 1999: 323)

Warning zeigt in einer Reihe von exemplarischen Textanalysen, wie sich Wissen und Imaginäres in literarischen Texten zueinander verhalten können. Besonders einschlägig ist hier Dantes Divina Commedia. In diesem Text wird eine abstrakte Wissensordnung, die sich u.a. aus der scholastischen Theologie speist, konkret ins Bild gesetzt. Dabei kommt es zum Widerstreit zwischen der aus dem theologischen Wissen heraus erklärbaren objektiven Jenseitsordnung, in der alle Seelen nach ihrem Tod an dem ihnen jeweils gebührenden Ort angesiedelt werden, d.h. einer statischen topologischen und topographischen Ordnung, in der Sujethaftigkeit (im Sinne von Lotman 1972: 333) ausgeschlossen ist, und der von den Seelen erinnerten und erzählten Diesseitsordnung, die geprägt ist durch sujethafte Grenzüberschreitungen. An diesen Sujets, so Warning, entzünde sich das Imaginäre der Commedia. Dieser Gegensatz wurde in der Danteforschung auch mit den Begriffen 'Sündenattribut' und 'poetisches Attribut' zu erfassen versucht. Im Gegensatz etwa zu Hugo Friedrich, der zwischen dem objektiven Sündenattribut und dem subjektiven poetischen Attribut eine klare Hierarchie zu erkennen glaubt (Friedrich 1942), argumentiert Warning, wie ich meine zu Recht, dass zwischen diesen beiden Typen von Attributen ein Spannungsverhältnis bestehe. Die Narration setzt eine Eigendynamik frei, an der sich das Imaginäre des Autors Dante, aber auch seiner Leser entfalten kann. Wenn etwa Odysseus in Inferno XXVI als ein Exempel augustinischer curiositas erscheint, so sind zwei Deutungsweisen möglich: 
Man kann mit ihr [sc. der curiositas] den Text ins Theologische zurückholen und damit poetisch stillstellen. Ebenso gut aber kann man in der curiositas genau jenen kritischen Punkt ausmachen, an dem Wissen ins Imaginäre umschlägt. Curiositas ist Wissenwollen jenseits des schon Gewußten, sie ist libidinös und damit imaginär besetztes Wissen, ein Wissenstrieb, der wie im Falle des Odysseus Grenzen des Gewußten überwinden will. (Warning 1999: 327)

Wenn nun aber Odysseus, dessen Geschichte exemplarisch für curiositas steht, sich an einem Jenseitsort befindet, an dem er offiziell für eine ganz andere Sünde bestraft wird, nämlich die, ein schlechter Ratgeber gewesen zu sein, dann kann man diese Diskrepanz mit Warning als ein Zeichen dafür deuten, dass sich der poetische Diskurs "in einen Konterdiskurs zu jenem Wissensdiskurs, dem sie [sc. die curiositas] entnommen ist" (ebd.), verwandelt. Die Ordnung der durch Fiktion modellierten Welt ist, so kann man Warnings These resümieren, nicht deckungsgleich mit der Ordnung der Wirklichkeit, wie sie durch die dominanten Diskurse einer Epoche vorgegeben wird. Da aber diesen dominanten Diskursen wie auch dem Gegendiskurs der Fiktion das radikal Imaginäre vorgelagert ist, situieren sich sowohl die epistemischen als auch die fiktionalen Diskurse in einem gemeinsamen Bezugsrahmen. Und dieser Rahmen lässt die Betrachtung literarisch-fiktionaler Texte in epistemischer Perspektive nicht nur als legitim, sondern sogar als notwendig erscheinen.

\subsection{Kendall L. Walton}

In seinem Buch Mimesis as Make-Believe entwickelt Kendall L. Walton eine Theorie der Fiktion, die als wesentlichen Bestandteil die Imagination enthält (Walton 1990). ${ }^{2}$ Sein Begriff der Fiktion beruht, wie schon der Titel seines Buches anzeigt, auf dem Konzept des makebelieve, welches er mit dem Spiel in Zusammenhang bringt. "Games of make-believe" sind für Walton Handlungen, die die Imagination zum Einsatz bringen oder erforderlich machen. Dabei werden normalerweise Auslöser, sogenannte props, benötigt. Dies können Puppen, Holzklötze oder andere Gegenstände sein. Diese Gegenstände werden beim Spiel mittels der Imagination in etwas anderes verwandelt, indem sich die Beteiligten vorstellen, dass sie dieses andere seien. Walton unterscheidet drei Typen von "games of make-believe": 1. das Spiel der Kinder, 2. Tagträume, 3. Fiktionen, die er auch als "representations" bezeichnet. Unter "works of fiction" versteht er "works whose function is to serve as props in games of make-believe" (Walton 1990: 72).

Was Waltons Ansatz leistet, ist, dass er, genau wie die Ansätze von Iser und Warning, die Fiktion nicht als etwas Negatives oder Defizitäres, sondern als etwas Positives zu fundieren versucht. Das in unserem Zusammenhang Entscheidende ist, dass er dabei die Imagination mit einbezieht. Die Imagination ist als wesentliches Vermögen des Menschen vorauszusetzen, damit Fiktion überhaupt entstehen kann. Der Baumstumpf oder der Busch im Wald, den spielende Kinder zu einem Bären erklären, kann überhaupt nur deshalb eine fiktionale Wirklichkeit konstituieren, weil die Imagination eine solche Transformation zulässt bzw. ermöglicht. Ebenso verhält es sich - und das ist Waltons entscheidende Erkenntnis - beim Betrachten von Kunstwerken, die eine fiktive Wirklichkeit darstellen. Beim Betrachten einer Statue oder eines Bildes wird dieser Gegenstand erst mittels der Imagination in das ver-

2 Die folgenden Überlegungen greifen zum Teil auf meine Studie Epistemologische Fiktionen (Klinkert 2010: 32-34) zurück. 
wandelt, was er darstellen soll. Denn es ist klar, dass zwischen dem materiellen Gegenstand - einer Statue oder einem Gemälde - und dem, was dieser materielle Gegenstand repräsentieren soll ("Napoleon", "Goethe" oder auch der "Wanderer über dem Nebelmeer"), ein gewaltiger Unterschied hinsichtlich des Materials, der Form, der Ausdehnung, der Eigenschaften usw. besteht. Dieser Unterschied kann nur überbrückt werden mithilfe der Imagination, die beim Betrachten einer Goethe-Statue ausblendet, dass es sich um einen zentnerschweren Gegenstand aus Stein oder Eisen handelt und nicht um einen realen Menschen, der wirklich gelebt hat.

Die Transformationsleistung der Imagination besitzt auch eine semiotische Dimension. Im Spiel kommt es nämlich zur regelhaften, imaginären Transformation von Gegenständen. So spricht Walton davon, dass es im Spiel nützlich sein kann, sich auf eine Spielregel ("initial stipulation or agreement", Walton 1990: 23) in Form einer Transformationsvorschrift (etwa "Let's call that stump a bear") zu verständigen, die es dann ermöglicht, unabhängig von der jeweiligen Gestalt des zu transformierenden Gegenstandes das Spiel in Gang zu halten:

Once the basic stipulation is made, further deliberation may be unnecessary; the characteristics of the stump may prompt all participants to imagine, nondeliberately, a large and ferocious bear rearing up on its hind legs, and each may confidently expect the others to imagine likewise. (Compare paintings and sculptures that depend heavily on their titles: the late Monets in the Musée Marmottan, Jacques Lipchitz's Reclining Nude with Guitar.) (Walton 1990: 23)

In der Walton'schen Konzeption ist also zu unterscheiden zwischen der Wirklichkeit, so wie sie ist - der Baumstumpf im Wald, die Puppe oder die Statue als materielles Objekt -, und der mittels der Imagination transformierten Wirklichkeit. Diese zweite Wirklichkeit, die auf dem Prinzip des make-believe beruht, kann man als Mimesis oder Fiktion bezeichnen. Damit beruht Waltons Ansatz - ganz ähnlich wie die Ansätze Isers und Warnings - auf einer dreistelligen Relation zwischen Wirklichkeit, Imagination und Fiktion: Wirklichkeit wird Walton zufolge mithilfe der Imagination in Fiktion verwandelt. (Bei Iser werden das Reale und das Imaginäre im Akt des Fingierens in eine zeichenhafte Interaktion gebracht, für Warning lassen sich wirklichkeitskonstituierende Diskurse des Wissens und der Gegendiskurs der Fiktion nur begreifen, wenn man sie auf ein beide fundierendes radikal Imaginäres bezieht.)

Wenn man sich nun auf die - bei Walton nicht allein im Mittelpunkt stehende - literarische Fiktion beschränkt, dann ist festzustellen, dass er in diesem Zusammenhang durchaus traditionell argumentiert, indem er zwischen Texten unterscheidet, die Fiktion generieren, und solchen, die auf Wirklichkeit verweisen. Hier fällt er gewissermaßen hinter die Innovativität seines eigenen Ansatzes zurück, etwa wenn er sagt:

It is not the function of biographies, textbooks, and newspaper articles, as such, to serve as props in games of make-believe. They are used to claim truth for certain propositions rather than to make propositions fictional. Instead of establishing fictional worlds, they purport to describe the real world. (Walton 1990: 70)

Zwar stimmt seine Analyse insofern, als es in der Tat pragmatische Rezeptionsbedingungen gibt, die dafür sorgen, dass man Biographien oder Zeitungsartikel nicht als Auslöser in "games of make-believe" verwendet, sie also nicht zum Gegenstand fiktionaler Kommunikation macht. Doch liegt hier nicht der entscheidende Punkt. Es ist nämlich zunächst und grundlegend $\mathrm{zu}$ fragen, welches die kognitiven Verarbeitungsprozesse sind, die bei der Lektüre eines Textes, sei dieser ein Zeitungsartikel oder ein Roman, stattfinden. Nimmt man diese Frage ernst, dann wird man kaum abstreiten können, dass jede Art von geschriebenem 
Text in vergleichbarer Weise die Imagination in Anspruch nimmt. Das heißt, die Imagination, welche den Text als materiellen Träger von Informationen in eine vorgestellte Wirklichkeit verwandelt, muss in jedem Fall vorausgesetzt werden, damit eine Rezeption erfolgreich sein kann. Selbstverständlich kommen bei solchen Rezeptionsprozessen dann auf einer sekundären Ebene die erlernten, in einer Kultur jeweils gültigen Regeln bezüglich Fiktionalität oder Referentialität zum Tragen. Die Kenntnis dieser Regeln erlaubt uns, einen Roman anders zu behandeln als einen Zeitungsartikel. Das ändert jedoch nichts an der grundlegenden Tatsache, dass in beiden Fällen die Imaginationsprozesse als apriorische Bedingungen der Semiose vorauszusetzen sind.

Daraus lässt sich die Schlussfolgerung ziehen, dass der auf Imagination basierende fiktionale Verarbeitungs- und Transformationsmodus der elementare ist, der bei jeder Form zeichengesteuerter Kommunikation zuallererst zum Tragen kommt. Auf einer weniger basalen Ebene erfolgt dann die Ausdifferenzierung verschiedener auf das Dargestellte bezogener Statuszuschreibungen wie real vs. fiktiv. Die Priorität des imaginationsbasierten Fiktionsmodus hat mit dem allen Zeichen konstitutiv eingeschriebenen Merkmal der Absenz zu tun. Ein Zeichen verweist stets auf etwas in ihm selbst nicht Vorhandenes. Der Zeichenbenutzer muss dieses Abwesende imaginär restituieren, um das Zeichen zu verstehen. Je komplexer das Zeichen, desto mehr Imaginationsarbeit muss geleistet werden, etwa bei Erzählungen. Aber auch wenn man lediglich ein Verkehrsschild wahrnimmt und interpretiert, wird bereits ein imaginationsgestützter Prozess in Gang gesetzt, dessen Dauer und Komplexität selbstverständlich mit denen anderer Prozesse, wie sie etwa beim Lesen von Dantes Divina Commedia im Spiel sind, nicht vergleichbar sind. Dennoch gilt, dass jeder zeicheninterpretierende Akt in seinem Kern ein fiktionaler ist, weil er sich auf einen Imaginationsvorgang stützt.

\section{Schlussbetrachtung}

Vergleichen wir abschließend kurz die drei vorgestellten Theorien. Ihnen allen ist gemeinsam, dass sie nicht auf der binären Opposition Wirklichkeit vs. Fiktion beruhen, sondern auf der dreistelligen Relation Wirklichkeit - Fiktion - Imagination/Imaginäres. Alle drei versuchen damit, Fiktion - in Abweichung von den 'Standardtheorien' - nicht negativ, sondern positiv zu bestimmen. Iser und Warning stellen auf den Konvergenzpunkt von Literatur und Wissen ab. Bei aller Unterschiedlichkeit der Begründungen und Argumentationen zeigt sich als Gemeinsamkeit, dass beide Autoren der Auffassung sind, dass die Literatur als eine Diskursform zu betrachten sei, in der das Imaginäre bearbeitet bzw. welche vom Imaginären gespeist werde. Diese Bearbeitung des Imaginären in der literarischen Fiktion sei gekoppelt an Prozesse des Wissens und der Erkenntnis. Iser denkt dabei im Grunde aristotelisch-mimetisch, wenn er sagt, dass in den Akten des Fingierens eine "Umformulierung formulierter Welt" stattfinde und dadurch Verstehbarkeit und Erfahrbarkeit ermöglicht würden. Warning dagegen argumentiert mit Foucault diskursarchäologisch, versucht allerdings die Episteme

wie auch die Fiktion mit Castoriadis im radikal Imaginären zu fundieren, und postuliert ein Hybridverhältnis zwischen literarischem und epistemischem Diskurs, welches erst auf einer Oberflächenebene durch Gattungsregeln desambiguiert werde. Walton argumentiert handlungstheoretisch, indem er Fiktion als eine Form des Spiels definiert. In seinem Fiktionskonzept spielt die Imagination als transformierendes Vermögen eine wichtige, wenn nicht die entscheidende Rolle. Man kann aus seiner Theorie ableiten, dass die Imagination die apriorische Voraussetzung für die Möglichkeit der Fiktion ist. Außerdem kann man, wenn man 
seine Ausführungen konsequent weiterdenkt, erkennen, dass bei der Rezeption von Texten unabhängig von deren fiktionalem oder referentiellem Status stets die Imagination zum Einsatz kommen muss und dass damit alle Formen der Zeicheninterpretation in ihrem Kern fiktional sind.

\section{Bibliographie}

Bundesverfassungsgericht 2007: Leitsätze zum Beschluss des Ersten Senats vom 13. Juni 2007 (1 BvR 1783/05): im Internet unter http://www.bundesverfassungsgericht.de/entscheidungen/rs20070613_1bvr178305.html [09.07.2014].

Castoriadis, Cornelius 1975: L'institution imaginaire de la société, Paris: Éd. du Seuil (Auflage 1999).

Foucault, Michel 1994 [1964]: “Un 'fantastique' de bibliothèque”, in: Daniel Defert \& François Ewald (eds.), Dits et écrits I (1954-1969), Paris: Gallimard: 293-325.

Foucault, Michel 1966: Les mots et les choses. Une archéologie des sciences humaines, Paris: Gallimard.

Foucault, Michel 1969: L'archéologie du savoir, Paris: Gallimard.

Friedrich, Hugo 1942: Die Rechtsmetaphysik der göttlichen Komödie. Francesca da Rimini, Frankfurt/M: Klostermann.

Genette, Gérard 1991: Fiction et diction, Paris: Éd. du Seuil.

Iser, Wolfgang 1991: Das Fiktive und das Imaginäre, Frankfurt/M: Suhrkamp (Auflage 1993).

Klinkert, Thomas 2010: Epistemologische Fiktionen. Zur Interferenz von Literatur und Wissenschaft seit der Aufklärung, Berlin / New York: de Gruyter.

Lotman, Jurij M. 1972: Die Struktur literarischer Texte, übers. v. Rolf-Dietrich Keil, München: Fink.

Petersen, Jürgen H. 2000: Mimesis - Imitatio - Nachahmung. Eine Geschichte der europäischen Poetik, München: Fink.

Platon 1998: Der Staat, übers. v. Otto Apelt, Hamburg: Meiner (unveränderter Nachdruck der Ausgabe Leipzig 1923).

Searle, John R. 2007 [1974/75]: "Der logische Status fiktionaler Rede”, in: Maria E. Reicher (ed.), Fiktion, Wahrheit, Wirklichkeit. Philosophische Grundlagen der Literaturtheorie, Paderborn: Mentis: 21-36.

Walton, Kendall L. 1990: Mimesis as Make-Believe. On the Foundations of the Representational Arts, Cambridge (Mass.) / London: Harvard Univ. Pr.

Warning, Rainer 1983: "Der inszenierte Diskurs. Bemerkungen zur pragmatischen Relation der Fiktion", in: Dieter Henrich \& Wolfgang Iser (eds.), Funktionen des Fiktiven, München: Fink: 183-205.

Warning, Rainer 1999: "Poetische Konterdiskursivität. Zum literaturwissenschaftlichen Umgang mit Foucault", in: ders., Die Phantasie der Realisten, München: Fink: 313-345. 


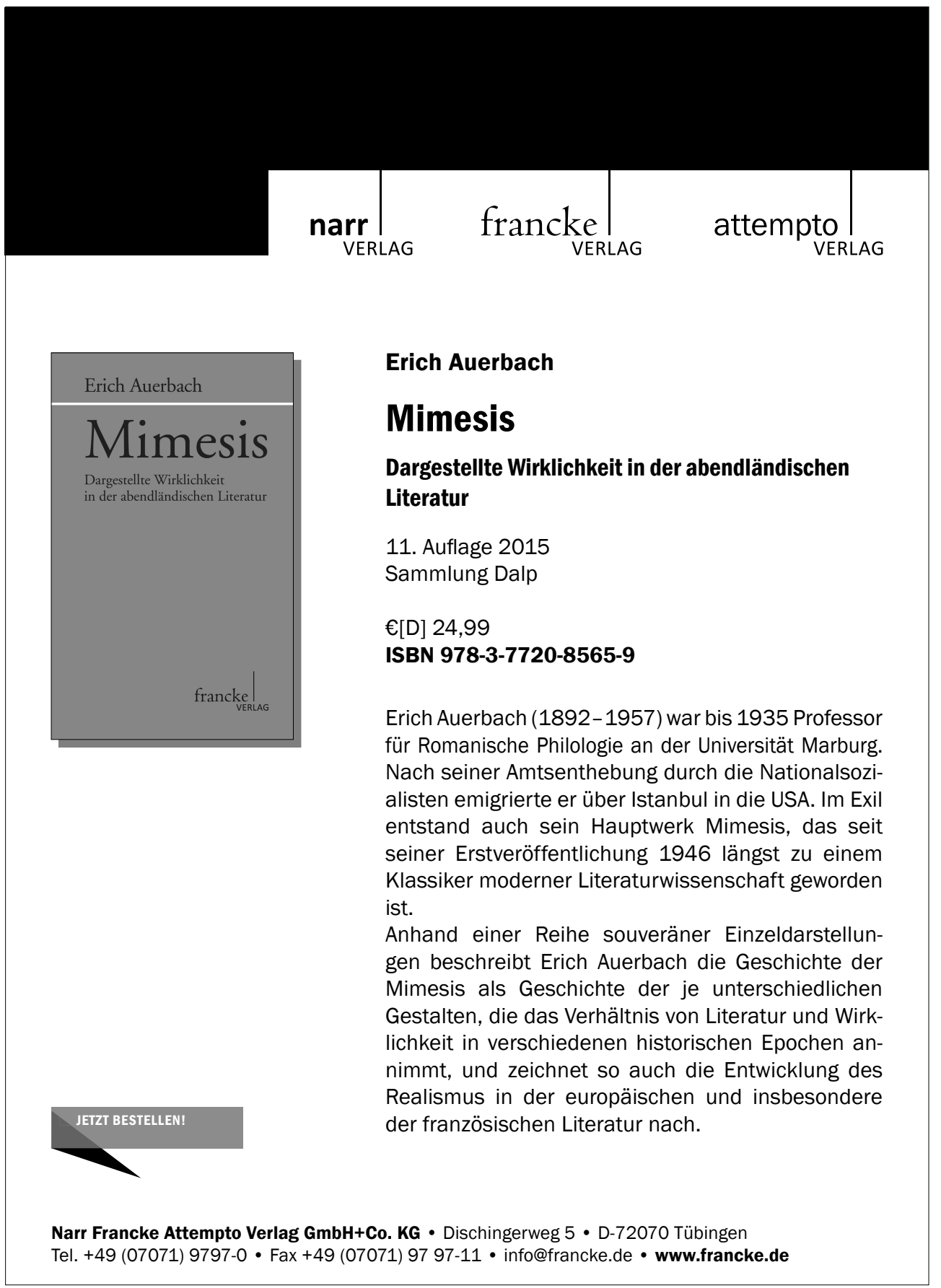

